\title{
Preterm deliveries affected by mechanical disadvantage in women with uterine myomas: a prospective cohort study from the Japan Environment and Children's Study
}

\author{
Tsuyoshi Murata ${ }^{1}$, Hyo Kyozuka ${ }^{1}$, Yuta Endo ${ }^{1}$, Toma Fukuda ${ }^{1}$, Shun Yasuda ${ }^{1}$, Akiko \\ Yamaguchi $^{1}$, Akiko Sato ${ }^{1}$, Yuka Ogata ${ }^{1}$, Kosei Shinoki ${ }^{1}$, Mitsuaki Hosoya ${ }^{1}$, Seiji \\ Yasumura $^{1}$, Koichi Hashimoto ${ }^{1}$, Hidekazu Nishigori ${ }^{1}$, and Keiya Fujimori ${ }^{1}$ \\ ${ }^{1}$ Fukushima Medical University
}

June 25, 2020

\begin{abstract}
Objective: To evaluate the incidence of adverse pregnancy outcomes (APOs) in women with uterine myomas and clarify the effect of uterine myomas on pregnancy outcomes. Design: Prospective cohort study Setting: The Japan Environment and Children's Study (between 2011-2014) Population: Women $(86,370)$ with singleton births after 22 weeks of gestation. Methods: Using logistic regression, the adjusted odds ratios (aORs) for APOs were calculated considering women without uterine myomas as the reference. Additionally, we used logistic regression to evaluate the effect of intrauterine infection (II) on the incidence of preterm births (PTB) and preterm premature rupture of membranes (pPROM). Main Outcome Measures: PTB (before 37 and 34 weeks), pPROM, II, and gestational hypertension (GH). Results: In women with uterine myomas, the aORs for PTB before 37 and 34 weeks, pPROM, II, and GH were 1.37 (95\% confidence interval [CI], 1.22-1.54), 1.61 (95\% CI, 1.27-2.05), 1.65 (95\% CI, 1.33-2.04), 1.05 (95\% CI, 0.75-1.46), and 1.20 (95\% CI, 1.05-1.38), respectively. In women with both uterine myomas and intrauterine infection, the aORs for PTB before 37 weeks and pPROM were not significantly increased. Conclusions: Intra-pregnancy uterine myomas were associated with an increased risk of APOs. II in women with uterine myomas was not associated with PTB or pPROM. These data suggest a potential mechanical disadvantage in pregnant women with uterine myomas. Funding: Ministry of the Environment, Japan Key words: uterine myoma, preterm birth, preterm premature rupture of membrane, intrauterine infection
\end{abstract}

\section{Introduction}

Uterine myomas (also known as leiomyomata, fibroids, fibromyomas, leiomyofibromas, and fibroleiomyomas) are benign tumors that originate from clonal proliferation of smooth muscle cells of the uterus; they are common among women of reproductive age, with a prevalence of $20-60 \% \cdot{ }^{1-4}$ The prevalence of uterine myomas in pregnant women has been reported as $0.4-10.7 \%$; however, conflicting evidence is available regarding the obstetric outcomes in this patient group. ${ }^{1,2}$ Although some studies have reported no increased risk of adverse pregnancy outcomes (APOs), ${ }^{5-7}$ other studies found that uterine myomas during pregnancy increased the risk of preterm births (PTB). ${ }^{1,8,9}$

In children, PTB accounts for $75 \%$ of perinatal mortality and more than half of long-term morbidity. ${ }^{10-12}$ PTB can occur spontaneously or may be induced medically. ${ }^{13}$ A major cause of spontaneous PTB is premature labor triggered because of unknown origin. ${ }^{13}$ Preterm premature rupture of membranes (pPROM), which is often followed by an intrauterine infection (II), is a risk factor associated with PTB. ${ }^{14,15}$ Gestational hypertension $(\mathrm{GH})$ is a common maternal complication and a risk factor for medically-induced PTB. ${ }^{12,13}$

PTB prevention regarding particular causal factors is paramount to improving neonatal outcomes; detailed 
evaluation of women at risk of PTB is required for tailored treatment. ${ }^{12}$ Therefore, accurate assessment of risk factors for PTB and its causal factors in women with uterine myomas are relevant to patients and physicians because of the frequency of uterine myomas. The majority of previous studies on PTB risks in women with uterine myomas were retrospective and involved small samples, ${ }^{7,16}$ yielding conflicting results on the association between uterine myomas and pPROM, II, and GH. ${ }^{1,2,5-8,17}$ Furthermore, the biochemical mechanisms of PTB in women with uterine myomas and of the effect of uterine myomas on the course of pregnancy remain unclear. ${ }^{1,2,8}$

Therefore, in the present study, we aimed to clarify the effects of uterine myomas on APOs; PTB, pPROM, II, and GH relative to the general population and to evaluate the effects of II on the incidence of PTB, and pPROM in women with uterine myomas. The goal was to clarify how uterine myomas affect APOs, using data from a nationwide Japanese prospective birth-cohort study.

\section{Methods}

\section{Study design}

This study was based on the data from the Japan Environment and Children's Study (JECS), which is a nationwide government-funded prospective birth cohort launched in January 2011 to investigate the effects of environmental factors on children's health. ${ }^{18,19}$ Briefly, the JECS is funded directly by Japan's Ministry of the Environment and involves the collaboration between the Programme Office (National Institute for Environmental Studies), Medical Support Centre (National Centre for Child Health and Development), and 15 regional centers (Hokkaido, Miyagi, Fukushima, Chiba, Kanagawa, Koshin, Toyama, Aichi, Kyoto, Osaka, Hyogo, Tottori, Kochi, Fukuoka, and South Kyushu/Okinawa). ${ }^{19}$ For inclusion as JECS participants, expectant mothers had to meet the following criteria: (1) residence within the study area at the time of recruitment and expectation to continue residing in Japan for the foreseeable future; (2) expected due date between August 1, 2011 and mid-2014; and (3) capacity to participate in the study without difficulty (i.e., ability to comprehend the Japanese language and complete a self-administered questionnaire).

There were two modes of recruitment: (1) at the time of the first prenatal examination at participating obstetric facilities; and (2) at local government offices issuing a pregnancy journal, called the Maternal and Child Health Handbook, given to all expecting mothers in Japan before they receive municipal services for pregnancy, delivery, and childcare. We contacted pregnant women through cooperating health care providers and/or local government offices issuing Maternal and Child Health Handbooks and registered those willing to participate. Self-administered questionnaires, which were completed by the women during the first trimester and second/third trimester, were used to collect information on demographic factors, medical history, physical and mental health, lifestyle, occupation, environmental exposure at home and in the workplace, housing conditions, and socioeconomic status. ${ }^{19}$

The JECS protocol was reviewed and approved by the Ministry of the Environment Institutional Review Board on Epidemiological Studies on March 23, 2010 (No. 15000141) ${ }^{18,19}$, and by the Ethics Committees of all participating institutions. The JECS was conducted in accordance with the Helsinki Declaration and other national regulations and guidelines. Written informed consent was obtained from all participating women.

\section{Data collection}

The current analysis used data released in June 2016 (data set: JECS-ag-20160424). Specifically, we used three types of data: (1) M-T1, which are data on maternal medical background, obtained using a selfreported questionnaire during the first trimester (the first questionnaire); (2) M-T2, which are data on partner lifestyle and socioeconomic status, obtained with a self-reported questionnaire during the second or third trimester (second questionnaire); (3) Dr-0m, which are data on obstetric outcomes such as gestational age and birth weight, collected throughout pregnancy and extracted from participants' medical records transcripts (provided by relevant institutions).

Participants with singleton pregnancies after 22 weeks of gestation were included in the present study. We 
excluded multiple pregnancies, abortions, stillbirths, and deliveries with unknown gestational age. Patients with uterine myomas were identified based on medical records transcripts.

Obstetric outcomes and confounding factors

PTB was classified into two categories-before 37 and 34 weeks-because PTB before 34 weeks required antenatal corticosteroid therapy for fetal maturation. ${ }^{20}$ pPROM was defined as spontaneous rupture of membranes before 37 weeks. II was identified clinically, which was derived from medical records transcripts. GH was defined as persistently elevated blood pressure ([?]140/90 $\mathrm{mmHg}$ ) after 20 weeks of pregnancy in an otherwise normotensive woman. ${ }^{21}$

The following items were analyzed as potential confounding factors: maternal age, maternal body mass index (BMI) before pregnancy, parity, maternal smoking status, maternal educational status, and annual household income. Participants were divided into three groups (maternal age $<20$ years, $20-34$ years, and [?]35 years). ${ }^{22,23}$ BMI before pregnancy was categorized into three groups $(<18.5,18.5-25.0$, and $<25.0)$. Parity was categorized into two groups (nulliparous and multiparous). Participants were requested to provide information about their smoking status by choosing one of the following: "kept smoking during pregnancy," "never smoked," "quit smoking before pregnancy," and "quit smoking during early pregnancy." Participants who chose "kept smoking during pregnancy" were included in the "smoking" category, while the other participants were included in the "non-smoking" category. Educational status was categorized into four groups based on the completed number of years of education (junior high school, $<10$ years; high school, 10-12 years; professional school or university, 13-16 years; and graduate school, [?]17 years). Annual household income was categorized into four levels $(<2,000,000,2,000,000-5,999,999,6,000,000-9,999,999$, and [?]10,000,000 JPY). These confounding factors were chosen based on clinical importance. ${ }^{24-26}$

\section{Statistical analysis}

Participants were stratified based on the presence of uterine myoma; clinical and demographic sample characteristics were reported accordingly. The Student's t-test was used to compare characteristics expressed as continuous variables between each group, and the chi-square test was used to compare characteristics expressed as categorical variables. First, adjusted odds ratios (aORs), and 95\% confidence intervals (CIs) for PTB, pPROM, II, and GH were calculated using a multiple logistic regression model with the control group as reference. Second, the effect of II on the incidence of PTB, and pPROM in women with, and without uterine myomas was evaluated using a multiple logistic regression model with women without II used as reference. All ORs were adjusted for maternal age, maternal BMI before pregnancy, parity, maternal smoking status, maternal educational status, and annual household income.

SPSS version 26 (IBM Corp., Armonk, NY, USA) was used for statistical analysis. A p-value $<0.05$ indicated statistical significance.

Role of the funding body

This work was supported by The Japan Environment and Children's Study which was funded by the Ministry of the Environment, Japan. The findings and conclusions of this article are solely the responsibility of the authors and do not represent the official views of the Ministry of the Environment, Japan.

\section{Results}

The total number of fetal records during 2011-2014 within the JECS was 104,102. A total of 86,370 participants met the inclusion criteria (Figure 1). Among them, 5,354 participants had uterine myomas (myoma group), while the remaining 81,016 were the participants without uterine myomas (control group).

Table 1 summarizes clinical and demographic characteristics, and obstetric outcomes. Except for II, the incidence of APOs was higher in the myoma group than in the control group.

The aORs of PTB before 37, and 34 weeks, pPROM, II, and GH in the myoma group were 1.37 (95\% confidence interval [CI], 1.22-1.54), 1.61 (95\% CI, 1.27-2.05), 1.65 (95\% CI, 1.33-2.04), 1.05 (95\% CI, 0.75- 
1.46), and 1.20 (95\% CI, 1.05-1.38), respectively. The aORs of PTB, pPROM, and GH were significantly higher in the myoma group than that in control group (Table 2).

The aORs of PTB and pPROM in women with II and uterine myomas were 1.81 (95\% CI, 0.64-5.15) and 1.29 (95\% CI, 0.17-9.54), while the aORs of PTB and pPROM in women with II without uterine myomas were 3.16 (95\% CI, 2.39-4.19) and 5.54 (95\% CI, 3.72-8.25), respectively. Although the aORs of PTB and pPROM were significantly increased in women with II without uterine myomas, the aORs were not significantly increased in women with II and uterine myomas (Table 3).

\section{Discussion}

\section{Main findings}

In the present study, there was a higher incidence of PTB, pPROM, and GH among pregnant women with uterine myomas than that among the control group. However, there was no significant association between uterine myomas during pregnancy and II. In addition, II was not associated with increased risk of PTB and pPROM in women with uterine myomas, contrary to the results in the general population. This suggests that PTB may be induced by mechanical factors in women with uterine myomas, rather than by II.

\section{Interpretation}

Uterine myomas during pregnancy increased the risk of PTB, which is consistent with findings of recent studies. ${ }^{1,2,8,9}$ A previous retrospective cohort study found that uterine myomas during pregnancy significantly increased PTB before 37 weeks (aOR, 1.5; 95\% CI, 1.3-1.8) and before 34 weeks (aOR, 1.4; 95\% CI, 1.01.8). ${ }^{1}$ However, another retrospective cohort study, in which the prevalence of uterine myomas was $1.5 \%$, and the risk estimates were based on only 183 women with uterine myomas, showed no such association. ${ }^{17}$ In contrast, the present study was based on a sample of 5,354 women with uterine myomas (uterine myomas prevalence of $6 \%$ ), which is comparable to the composition of recent studies. ${ }^{1,2}$ Overall, these findings support the hypothesis that uterine myomas during pregnancy increase the risk of PTB.

The risk of pPROM in the myoma group was greater than that in the control group. Previous studies on this association produced conflicting results. ${ }^{2,5-8,17}$ One retrospective cohort study reported higher incidence of pPROM (aOR, 1.3; 95\% CI, 1.0-1.7) ${ }^{1}$; our findings are consistent with those of that study. Because pPROM precedes $40-50 \%$ of PTB cases, ${ }^{27,28}$ it is also likely to induce PTB in women with uterine myomas. Because the rate of neonatal mortality, and morbidity associated with PTB is higher in groups affected by pPROM than in any other subgroup of $\mathrm{PTB},{ }^{28} \mathrm{pPROM}$ is likely to increase the risk of adverse neonatal outcomes in women with uterine myomas. These findings suggest that obstetricians should communicate the risk of pPROM and PTB to pregnant women with uterine myomas.

Compared to the control group, we found no increased risk of II in the myoma group; additionally, II did not increase the risk of PTB, or pPROM in the myoma group. No study to-date has shown any association between uterine myomas and increased risk of II in pregnancy. Previous studies have shown II to be a major risk factor for $\mathrm{PTB}^{13}$ and pPROM prior to labor. ${ }^{27}$ Moreover, microbiological studies suggested that II might account for $25-40 \%$ of PTB cases, while approximately $70 \%$ of pPROM cases were associated with II. ${ }^{13}$ The present study showed that II was a significant risk factor of PTB, and pPROM in the control group, but not in the myoma group. Although the sample size with II was small, and there were ambiguities regarding the classification and diagnosis of II owing to confusion of clinical characteristics, biomarkers, and pathological findings, ${ }^{29}$ the present study was the first large cohort analysis to evaluate the association between uterine myomas during pregnancy and II.

We found that the risk of GH was greater in the myoma group than in the control group. Other evidence on this association is conflicting, ${ }^{2,5-8,17}$ and the etiology of GH in women with uterine myomas is unknown. Although GH is a risk factor for PTB, the underlying mechanism of its effect on PTB remains unclear, as this study did not evaluate the mechanism of PTB. Concurrently, while we defined GH as only elevated maternal blood pressure, we did not account for hypertensive disorders of pregnancy (HDP) in the present analysis, because subtypes of HDP differ in characteristics, ${ }^{21,30}$ and extraction of HDP in the present data set with 
lacking laboratory data may lead to inaccurate analysis. Because of these unclarified issues, future studies should consider the specific mechanisms of PTB and differentiate between GH and HDP. Nevertheless, our findings suggested that maternal complications such as GH might be risk factors for PTB in women with uterine myomas.

\section{Strengths and limitations}

A major strength of the present study is use of a large sample of a nationwide cohort study that yields robust findings regarding the impact of uterine myomas on APOs. The presented aORs provide estimates of risk of APOs associated with uterine myomas in pregnancy. Specifically, the aOR of PTB before 34 weeks in women with uterine myomas was nearly twice that of the control group, suggesting that uterine myomas during pregnancy might be directly linked to neonatal outcomes. The present findings suggest the need to appropriately evaluate the PTB risk and manage pregnancies in women with uterine myomas for suitable PTB prevention, and improve neonatal outcomes, including administration of vaginal progesterone, antenatal corticosteroids, antibiotics, and magnesium sulfate. ${ }^{31-33}$

The present findings suggest a characteristic etiology of PTB, and pPROM in women with uterine myomas. Although the biological mechanism of PTB induced by uterine myomas remains unclear, ${ }^{1,8}$ studies have shown that spontaneous PTB including pPROM in cases with uterine myomas was associated with distortion of the uterine cavity and loss of uterine distensibility. ${ }^{34,35}$ In addition, hormonal changes have been implicated in spontaneous PTB. ${ }^{2}$ Uterine myomas may compromise the myometrium and cause decidualization of endometrial stromal fibroblasts, inducing spontaneous PTB, as is the case in endometriosis. ${ }^{2,36,37}$ Therefore, the present findings support the notion of a mechanical mechanism of uterine myomas rather than one depending on inflammatory factors in PTB and pPROM. Clinically, PTB, and pPROM without II may lack clinical features and may lead to occult incidence of PTB, and pPROM.

The present study has several limitations. First, the protocol for diagnosing uterine myomas in early stage of pregnancy was not unified, and variations in their number, size, and site, as well as history of myomectomy were not considered. As these factors may affect obstetric outcomes, ${ }^{2,38}$ future studies should include evaluations of these factors. Nevertheless, this may support the notion that uterine myomas by itself increase APOs regardless of number, size, and location. Second, several maternal characteristics previously associated with PTB were not considered, i.e. certain demographic and psychological characteristics, history of PTB in detail, adverse behaviors, uterine contractions, cervical length, and biological and genetic markers. These implicated factors of $\mathrm{PTB}^{39}$ should be evaluated in future studies. Nevertheless, we included a large sample and accounted for factors such as smoking status, maternal educational status, and annual household income, all of which contribute to the robustness of the present findings.

\section{Conclusion}

Uterine myomas during pregnancy significantly increase the incidence of PTB, pPROM, and GH, regardless of number, size and localization. PTB and pPROM in women with uterine myomas were not associated with II, suggesting a mechanical disadvantage and the risk of occult PTB and pPROM in this patient group. Obstetricians should counsel their patients regarding the risks associated with uterine myomas in pregnancy and offer suitable interventions to prevent and manage APOs.

Acknowledgements: The findings and conclusions of this article are solely the responsibility of the authors and do not represent the official views of the Ministry of the Environment, Japan. The authors are grateful to all participants of the study.

Members of the JECS Group as of 2020: Michihiro Kamijima (principal investigator, Nagoya City University, Nagoya, Japan), Shin Yamazaki (National Institute for Environmental Studies, Tsukuba, Japan), Yukihiro Ohya (National Center for Child Health and Development, Tokyo, Japan), Reiko Kishi (Hokkaido University, Sapporo, Japan), Nobuo Yaegashi (Tohoku University, Sendai, Japan), Koichi Hashimoto (Fukushima Medical University, Fukushima, Japan), Chisato Mori (Chiba University, Chiba, Japan), Shuichi Ito (Yokohama City University, Yokohama, Japan), Zentaro Yamagata (University of Yamanashi, Chuo, Japan), Hidekuni 
Inadera (University of Toyama, Toyama, Japan), Takeo Nakayama (Kyoto University, Kyoto, Japan), Hiroyasu Iso (Osaka University, Suita, Japan), Masayuki Shima (Hyogo College of Medicine, Nishinomiya, Japan), Youichi Kurozawa (Tottori University, Yonago, Japan), Narufumi Suganuma (Kochi University, Nankoku, Japan), Koichi Kusuhara (University of Occupational and Environmental Health, Kitakyushu, Japan), and Takahiko Katoh (Kumamoto University, Kumamoto, Japan).

Disclosure of Interests: The authors report no conflicts of interest.

Contribution to Authorship: All authors approved the final manuscript. T.M. initiated study concept and design. T.M., H.K., Y.E., T.F., S.Y., A.Y., K.H., H.N., and K.F. contributed to study design. K.S., A.S., and Y.O. collected the data. T.M. analyzed the data and wrote the manuscript. M.H., S.Y., K.H., K.S., A.S., Y.O., H.N., K.F., and the JECS group reviewed the manuscript and provided critical advice.

Details of Ethics Approval: The JECS protocol was reviewed and approved by the Ministry of the Environment Institutional Review Board on Epidemiological Studies on March 23, 2010 (No. 15000141) 18,19 and by the Ethics Committees of all participating institutions. The JECS was conducted in accordance with the Helsinki Declaration and other national regulations and guidelines. Written informed consent was obtained from all participating women.

Funding: The Japan Environment and Children's Study was funded by the Ministry of the Environment, Japan. The findings and conclusions of this article are solely the responsibility of the authors and do not represent the official views of the Ministry of the Environment, Japan.

\section{References}

[1] Stout MJ, Odibo AO, Graseck AS, Macones GA, Crane JP, Cahill AG. Leiomyomas at routine secondtrimester ultrasound examination and adverse obstetric outcomes. Obstet Gynecol. 2010 Nov;116(5):105663.

[2] Girault A, Le Ray C, Chapron C, Goffinet F, Marcellin L. Leiomyomatous uterus and preterm birth: an exposed/unexposed monocentric cohort study. Am J Obstet Gynecol. 2018 Oct;219(4):410.e1-7.

[3] Vlahos NF, Theodoridis TD, Partsinevelos GA. Myomas and adenomyosis: Impact on reproductive outcome. Biomed Res Int. 2017;2017:5926470.

[4] Saleh HS, Mowafy HE, Hameid AAAE, Sherif HE, Mahfouz EM. Does uterine fibroid adversely affect obstetric outcome of pregnancy? Biomed Res Int. 2018;2018:8367068.

[5] Coronado GD, Marshall LM, Schwartz SM. Complications in pregnancy, labor, and delivery with uterine leiomyomas: A population-based study. Obstet Gynecol. 2000 May;95(5):764-9.

[6] Exacoustòs C, Rosati P. Ultrasound diagnosis of uterine myomas and complications in pregnancy. Obstet Gynecol. 1993 Jul;82(1):97-101.

[7] Davis JL, Ray-Mazumder S, Hobel CJ, Baley K, Sassoon D. Uterine leiomyomas in pregnancy: A prospective study. Obstet Gynecol. 1990 Jan;75(1):41-4.

[8] Qidwai GI, Caughey AB, Jacoby AF. Obstetric outcomes in women with sonographically identified uterine leiomyomata. Obstet Gynecol. 2006 Feb;107(2 Pt 1):376-82.

[9] Chen YH, Lin HC, Chen SF, Lin HC. Increased risk of preterm births among women with uterine leiomyoma: a nationwide population-based study. Hum Reprod. 2009 Dec;24(12):3049-56.

[10] McCormick MC. The contribution of low birth weight to infant mortality and childhood morbidity. N Engl J Med. 1985 Jan;312(2):82-90.

[11] Wen SW, Smith G, Yang Q, Walker M. Epidemiology of preterm birth and neonatal outcome. Semin Fetal Neonatal Med. 2004 Dec;9(6):429-35. 
[12] López Bernal A. Overview. Preterm labour: Mechanisms and management. BMC Pregnancy Childbirth. 2007 Jun;7 Suppl 1 (Suppl 1):S2.

[13] Goldenberg RL, Culhane JF, Iams JD, Romero R. Epidemiology and causes of preterm birth. Lancet. 2008 Jan;371(9606):75-84.

[14] Ananth CV, Vintzileos AM. Epidemiology of preterm birth and its clinical subtypes. J Matern Fetal Neonatal Med. 2006 Dec;19(12):773-82.

[15] Romero R, Quintero R, Oyarzun E, Wu YK, Sabo V, Mazor M, Hobbins JC. Intraamniotic infection and the onset of labor in preterm premature rupture of the membranes. Am J Obstet Gynecol. 1988 Sep;159(3):661-6.

[16] Rice JP, Kay HH, Mahony BS. The clinical significance of uterine leiomyomas in pregnancy. Am J Obstet Gynecol. 1989 May;160(5 Pt 1):1212-6.

[17] Vergani P, Ghidini A, Strobelt N, Roncaglia N, Locatelli A, Lapinski RH, et al. Do uterine leiomyomas influence pregnancy outcome? Am J Perinatol. 1994 Sep;11(5):356-8.

[18] Kawamoto T, Nitta H, Murata K, Toda E, Tsukamoto N, Hasegawa M, et al. Rationale and study design of the Japan Environment and Children's Study (JECS). BMC Public Health. 2014 Jan;14:25.

[19] Michikawa T, Nitta H, Nakayama SF, Yamazaki S, Isobe T, Tamura K, et al. Baseline profile of participants in the Japan Environment and Children's Study (JECS). J Epidemiol. 2018;28(2):99-104.

[20] Committee on Obstetric Practice. Committee Opinion No. 713: Antenatal corticosteroid therapy for fetal maturation. Obstet Gynecol. 2017 Aug;130(2):e102-9.

[21] Brown MA, Magee LA, Kenny LC, Karumanchi SA, McCarthy FP, Saito S, et al. Hypertensive disorders of pregnancy: ISSHP classification, diagnosis, and management recommendations for international practice. Hypertension. 2018 Jul;72(1):24-43.

[22] Kyozuka H, Fujimori K, Hosoya M, Yasamura S, Yokoyama T, Sato A, et al. The effect of maternal age at the first childbirth on gestational age and birth weight: The Japan Environment and Children's Study (JECS). J Epidemiol. 2019 May;29(5):187-91.

[23] Kyozuka H, Fujimori K, Hosoya M, Yasumura S, Yokoyama T, Sato A, et al. The Japan Environment and Children's Study (JECS) in Fukushima Prefecture: Pregnancy outcome after the great East Japan earthquake. Tohoku J Exp Med. 2018 Sep;246(1):27-33.

[24] Luke B, Brown MB. Evaluated risks of pregnancy complications and adverse outcomes with increased maternal age. Hum Reprod. 2007 May;22(5):1264-72.

[25] Suzuki K, Shinohara R, Sato M, Otawa S, Yamagata Z. Association between maternal smoking during pregnancy and birth weight: an appropriately adjusted model from the Japan Environment and Children's Study. J Epidemiol. 2016 Jul;26(7):371-7.

[26] Ancel PY. Preterm labor: pathophysiology, risk factors and outcomes. J Gynecol Obstet Biol Reprod (Paris). 2002 Nov;31(7 Suppl):5S10-21. [In French]

[27] Naeye RL, Peters EC. Causes and consequences of premature rupture of fetal membranes. Lancet. 1980 Jan;1(8161):192-4.

[28] Menon R, Richardson LS. Preterm prelabor rupture of the membranes: A disease of the fetal membranes. Semin Perinatol. 2017 Nov;41(7):409-19.

[29] Higgins RD, Saade G, Polin RA, Grobman WA, Buhimschi IA, Watterberg K, et al. Evaluation and management of women and newborns with a maternal diagnosis of chorioamnionitis: Summary of a workshop. Obstet Gynecol. 2016 March;127(3):426-36. 
[30] American College of Obstetricians and Gynecologists; Task Force on Hypertension in Pregnancy. Hypertension in pregnancy. Report of the American College of Obstetricians and Gynecologists' Task Force on Hypertension in Pregnancy. Obstet Gynecol. 2013 Nov;122(5):1122-31.

[31] Roberts D, Brown J, Medley N, Dalziel SR. Antenatal corticosteroids for accelerating fetal lung maturation for women at risk of preterm birth. Cochrane Database Syst Rev. 2017 Mar;3(3):CD004454.

[32] Jarde A, Lutsiv O, Beyene J, McDonald SD. Vaginal progesterone, oral progesterone, 17-OHPC, cerclage, and pessary for preventing preterm birth in at-risk singleton pregnancies: An updated systematic review and network meta-analysis. BJOG. 2019 Apr;126(5):556-67.

[33] Duley L, Gülmezoglu AM, Henderson-Smart DJ, Chou D. Magnesium sulphate and other anticonvulsants for women with pre-eclampsia. Cochrane Database Syst Rev. 2010 Nov;2010(11):CD000025.

[34] Shavell VI, Thakur M, Sawant A, Kruger ML, Jones TB, Singh M, et al. Adverse obstetric outcomes associated with sonographically identified large uterine fibroids. Fertil Steril. 2012 Jan;97(1):107-10.

[35] Marzano S, Padula F, Meloni P, Anceschi MM. Preterm delivery at low gestational age: risk factors for short latency. A multivariated analysis. J Prenat Med. 2008 Apr;2(2):15-8.

[36] Zullo F, Spagnolo E, Saccone G, Acunzo M, Xodo S, Ceccaroni M, et al. Endometriosis and obstetrics complications: A systematic review and meta-analysis. Fertil Steril. 2017 Oct;108(4):667-672.e5.

[37] Petraglia F, Arcuri F, de Ziegler D, Chapron C. Inflammation: A link between endometriosis and preterm birth. Fertil Steril. 2012 Jul;98(1):36-40.

[38] Ciavattini A, Clemente N, Delli Carpini G, Di Giuseppe J, Giannubilo SR, Tranquilli AL. Number and size of uterine fibroids and obstetric outcomes. J Matern Fetal Neonatal Med. 2015 Mar;28(4):484-8.

[39] Goldenberg RL, Goepfert AR, Ramsey PS. Biochemical markers for the prediction of preterm birth. Am J Obstet Gynecol. 2005 May;192(5 Suppl):S36-46.

Table/Figure caption List

Table 1. Demographic and clinical characteristics and obstetric outcomes of participants stratified according to the uterine myoma status

SD: standard deviation, BMI: body mass index, PTB: preterm births, pPROM: preterm premature rupture of membranes, II: intrauterine infection, GH: gestational hypertension.

Table 2. Adjusted odds ratios (aORs) for obstetric complications in the myoma group

PTB: preterm births, pPROM: preterm premature rupture of membranes, II: intrauterine infection, GH: gestational hypertension, Ref: reference.

The multivariate logistic regression analyses were adjusted for maternal age, body mass index before pregnancy, parity, maternal smoking status, maternal educational status, and annual household income

Table 3. Adjusted odds ratios (aORs) for PTB and pPROM in women with II

PTB: preterm births, pPROM: preterm premature rupture of membrane, II: intrauterine infection, Ref: reference.

The multivariate logistic regression analyses were adjusted for maternal age, body mass index before pregnancy, parity, maternal smoking status, maternal educational status, and annual household income.

Figure 1. Flow chart depicting participant eligibility for study enrollment

\section{Hosted file}


Table 1.docx available at https://authorea.com/users/327152/articles/462511-pretermdeliveries-affected-by-mechanical-disadvantage-in-women-with-uterine-myomas-aprospective-cohort-study-from-the-japan-environment-and-children-s-study

\section{Hosted file}

Table 2.docx available at https://authorea.com/users/327152/articles/462511-pretermdeliveries-affected-by-mechanical-disadvantage-in-women-with-uterine-myomas-aprospective-cohort-study-from-the-japan-environment-and-children-s-study

\section{Hosted file}

Table 3.docx available at https://authorea.com/users/327152/articles/462511-pretermdeliveries-affected-by-mechanical-disadvantage-in-women-with-uterine-myomas-aprospective-cohort-study-from-the-japan-environment-and-children-s-study

\section{Hosted file}

Figure 1.docx available at https://authorea.com/users/327152/articles/462511-pretermdeliveries-affected-by-mechanical-disadvantage-in-women-with-uterine-myomas-aprospective-cohort-study-from-the-japan-environment-and-children-s-study 